\title{
RELAÇÃo ENTRE A DESIGUALDADE E EDUCAÇÃo NO BRASIL: UMA ESTIMATIVA DE DADOS EM PAINEL (1995-2009)
}

\author{
Maria Iná de Abreu Souza' \\ Fernando Henrique Taques² \\ Jailson da Conceição Teixeira de Oliveira ${ }^{3}$ \\ Douglas Alcântara Alencar ${ }^{4}$
}

\section{Resumo}

A literatura que versa sobre capital humano argumenta que a educação possui um efeito positivo na produtividade do fator trabalho, o que resulta em aumento no nível de salários. Com efeito, coeteris paribus, o aumento da escolaridade reflete no aumento do produto e pode contribuir na redução na desigualdade. Nesse contexto, o objetivo deste trabalho é investigar se há uma relação inversa entre a desigualdade de renda e educação para as Unidades Federativas do Brasil no período de 1995 e 2009. Para tanto, parte da metodologia econométrica de dados de painel com indicadores de desigualdade de renda e educação, além da renda domiciliar per capita. Os resultados obtidos vão no mesmo sentido que diversas evidências sobre o tema ao indicarem que as variáveis renda e educação são inversamente correlacionadas com a desigualdade de renda, sendo os resultados estatisticamente significantes.

Palavras-chave: Educação; Renda e Desigualdade.

Classificação JEL: I24, D63.

Graduada em Ciências Econômicas pelas Faculdades Metropolitanas Unidas (FMU).

2 Mestre em Economia pelo PEPEP-PUC/SP e Professor das Faculdades Metropolitanas Unidas (FMU) e do Serviço Nacional de Aprendizagem Comercial (SENAC/SP).

3 Professor do Departamento de Economia da Universidade Federal da Paraíba e Doutorando do Programa de PósGraduação em Economia pelo PPGE/UFPB.

4 Doutorando em Economia pelo Centro de Desenvolvimento e Planejamento Regional (CEDEPLAR)/UFMG, pesquisador Associado do Departamento de Economia - UNESP Araraquara e Professor das Faculdades Metropolitanas Unidas (FMU). 


\section{INTRODUÇÃO}

As abordagens de Schultz (1963) e posteriormente Becker (1964), revelam que o investimento em capital humano pode contribuir positivamente sobre o nível de renda. Nessa ótica, a educação é caracterizada como um investimento, pois possui capacidade de gerar aumento da produtividade do fator trabalho. Como consequência, uma melhora distributiva no nível educacional pode refletir em efeitos positivos sobre o nível de desigualdade de renda de uma população.

Para França, Gasparini e Loureiro (2005), a importância da educação tem sido reconhecida e ressaltada como fator de crescimento econômico de um país por sua associação com a produtividade do fator trabalho, dentro da teoria do capital humano. Maior produtividade representa maior eficiência econômica, portanto, maior geração de produto por unidade de fator de produção e, por consequência maior nível de produto. $\mathrm{O}$ aumento da produtividade do trabalho, ao mesmo tempo em que contribui para antecipar o crescimento econômico, tem também o efeito de redistribuir de maneira mais igualitária a renda porque aumenta a remuneração dos salários, fato de particular importância nos países em desenvolvimento, onde eles são em geral mais baixos.

Jallade (1978) vai além e assegura que políticas públicas voltadas para o investimento em educação podem promover uma melhora na distribuição de renda. Assim, tal mecanismo pode ser dado sob a forma financiamento do ensino superior (bolsas de estudo, por exemplo), investimento físico (estrutura) ou mesmo advindo de natureza intelectual.

Essa perspectiva de análise pautada no capital humano suscitou também a elaboração de pesquisas sobre o tema para o Brasil. Fishlow (1972) e Langoni (1973) partem de trabalhos seminais sobre o tema e concluem que a educação possui um papel importante na determinação da trajetória da desigualdade de renda. Posteriormente, trabalhos como de Velloso (1979), Ballasiano, Seabra e Lemos (2005), Menezes Filho e Oliveira (2014), Salvato, Ferreira e Duarte (2010) e Barros, Franco e Mendonça (2007) também tratam da relação entre desigualdade de renda e educação.

Assim, partindo dessa contextualização, é de interesse testar a hipótese se o aumento médio de anos de estudos implica em uma melhor condição distributiva da renda, tal como versa a perspectiva de capital humano. 
Cabe mencionar que o Brasil é um país caracterizado não apenas por apresentar desigualdades em termos de renda, mas também de ensino. Dados do Instituto de Pesquisas Econômicas Aplicadas (IPEA) indicam que a educação média (anos de estudo) do brasileiro aumentou consideravelmente desde a década de 1980, todavia ainda persiste uma desigualdade entre as unidades federativas. Estados associados a uma renda domiciliar per capita média menor geralmente também apresentam um menor nível de educação.

Assim, o objetivo dessa pesquisa é analisar a relação existente entre desigualdade de renda e educação para o Brasil. Para tanto, parte da metodologia de dados em painel (efeitos fixos e aleatórios) para dados das unidades federativas do Brasil (além do Distrito Federal) no período entre 1995 e 2009.

O trabalho está dividido em três seções, além desta introdução e da conclusão. A primeira parte apresenta o referencial teórico presente na literatura de capital humano. Na segunda parte é realizada uma revisão sobre estudos relativos ao Brasil. Na terceira parte são descritos os dados e a metodologia, bem como são reportados os resultados das estimativas econométricas.

\section{A RELAÇÃO ENTRE EDUCAÇÃO E DESIGUALDADE DE RENDA}

\subsection{Aspectos Teóricos}

Becker (1964) formula a teoria do capital humano a partir da ideia que o agente econômico faz uma relação de custo-benefício na tomada de decisão. Então, analisa o investimento em seu nível educacional a partir dos benefícios que espera obter posteriormente e os custos envolvidos no processo de aprendizado (tanto o desembolso voltado à educação quanto ao custo de oportunidade relativo ao tempo para dedicação a tal escolha).

A formulação do argumento segue com uma divisão entre dois tipos possíveis de formação educacional dos agentes: geral e específica. A primeira implica em uma formação associada ao ganho de produtividade do trabalho que, na média, tende a elevar a produtividade da população como um todo e, portanto, implica no crescimento da economia. Porém, segundo o autor, reside nesse contexto um problema de informação assimétrica, pois o custo 
decorrente dessa formação deve caber ao próprio agente, uma vez que as empresas não possuem incentivos para investir na formação do funcionário, pois com o maior nível de escolaridade o funcionário poderia migrar para outras empresas que forneçam salários mais atrativos. Deste modo, caberia ao governo ou a outras fontes o financiamento do capital humano, ou mesmo ao próprio agente (BECKER, 1964).

Por outro lado, segundo o autor, o investimento na formação específica deve ser realizado total ou parcialmente pelas empresas e direcionado unicamente àqueles funcionários cuja relação de trabalho é durável, uma vez que essa poderá usufruir dos benefícios decorrentes desse investimento em termos de produtividade do capital humano de seus funcionários. $\mathrm{O}$ argumento é válido inclusive para trabalhos que requerem mão de obra especializada e muitas vezes carecem de investimentos específicos para formação do funcionário. De maneira análoga:

\footnotetext{
De acordo com a teoria de distribuição pessoal da renda, os agentes econômicos escolhem, a partir de suas preferências individuais e dos e dos retornos de mercado dos diversos níveis educacionais, alocar seu tempo na educação, como forma de maximizar o valor presente do bem-estar ao longo dos ciclos de vida (TRINDADE, 2010, pg. 11).
}

Para Fernandes (2001) o investimento em capital humano, tratando esse conceito como sendo a escolarização ou a qualificação de um indivíduo, seria o meio pelo qual os indivíduos podem visualizar crescimentos potenciais em seus rendimentos futuros. Levando em consideração que durante o ciclo de vida de uma pessoa espera-se que ele se dedique integralmente à escolarização, então quanto maior a dedicação à educação formal e a qualquer outra forma de qualificação, maior será o estoque de capital humano, mantendo constante o seu estoque inicial de capital.

Velloso (1979), por sua vez, argumenta que o investimento em treinamento ou mesmo em capital humano como, por exemplo, a frequência à escola acarreta custos, tanto em termos de despesas diretas quanto em termos de custos de oportunidades. Portanto, supondo que as habilidades adquiridas com a educação levem a uma produtividade maior e se os salários equivalem ao valor do produto marginal do trabalhador, então um 
acréscimo de educação resulta em renda mais elevada. Assim, o estoque de educação incorporado a um indivíduo é uma fonte de capital humano. Empiricamente a educação tem sido frequentemente considerada como a principal fonte de capital humano.

Segundo Pereira (2001), os investimentos em educação resultam em dois tipos de efeitos: sociais e privados. Os efeitos sociais seriam as externalidades decorrentes da educação de um indivíduo, ou seja, os benefícios adquiridos pelos demais decorrentes de seu conhecimento ou habilidade. Já o efeito privado decorre de uma elevação do nível de educação que implica um impacto exclusivamente sobre os indivíduos, tendo um aumento da sua capacidade produtiva e de um melhor estoque de qualificação, isso corresponde ao aumento salarial decorrente da maior produtividade e de melhor capacidade de adaptação ao meio em que ele convive. Um exemplo seria o reconhecimento das empresas, onde pessoas com maior nível de escolaridade podem ser treinadas com menor custo. Há vários efeitos de caráter privado, como o aumento da qualidade de vida advindo de uma utilização mais eficiente dos recursos familiares e da melhor compreensão das informações sobre saúde, nutrição entre outros impactos, que podem reduzir o tamanho das famílias e diminuição da pobreza.

Barros, Henrique e Mendonça (2002) esclarecem que a contribuição da educação (capital humano) para a desigualdade salarial (remuneração) é função de dois fatores. Primeiro, ela depende do nível de desigualdade educacional. Assim, quanto maior a heterogeneidade da força de trabalho, maior o nível de desigualdade salarial. Segundo os autores, a desigualdade no salário é dependente também de como o mercado de trabalho traduz a desigualdade educacional em desigualdade salarial, isto é, qual o valor monetário que o mercado de trabalho atribui a cada ano adicional de escolaridade. Desse modo, quanto mais alto for esse valor, maior será a desigualdade salarial associada a cada nível de desigualdade educacional.

Para explicar o processo de geração e reprodução da desigualdade, Barros e Mendonça (1995), elaboraram um modelo teórico baseado numa sequencia de corridas (ciclo de vida de uma geração) em que cada corrida é formada por três elementos: a) um conjunto de participantes, cada um com um volume de recursos; b) um total de prêmios e c) um conjunto de regras. Nesta corrida, as regras e os prêmios podem ser distintos. As regras estabe- 
lecem fatores como, por exemplo, a forma como os participantes devem se comportar; a forma como a produtividade dos participantes será avaliada; e a forma como o total de prêmios será dividido entre os participantes de acordo com o seu desempenho.

No modelo relacionado à corrida, o volume inicial de recurso de cada participante depende de seus antepassados nas corridas anteriores. Para Barros e Mendonça (1995), a corrida se constitui em duas etapas. A primeira está relacionada ao período da infância e a adolescência, nesta etapa os participantes acumulam o capital humano e se preparam para a competição. Há três tipos de recursos para preparação destes participantes: habilidades, recursos públicos e recursos privados. Na segunda etapa ocorre a competição no mercado de trabalho.

Haverá desigualdade, afirmam Barros e Mendonça (1995), na competição do final da primeira etapa na preparação do participante, de acordo com a heterogeneidade dos participantes quanto ao volume de capital humano acumulado. A heterogeneidade é denominada desigualdade de condições. No final da segunda etapa, haverá também uma desigualdade de resultados tornando os prêmios desiguais.

Analisam ainda três situações para descrever o processo de geração de desigualdade. Para primeira situação, o grau de preparação de cada participante é determinado apenas pelas características natas, visto que há recursos públicos ou privados. Na segunda situação, em que se consideram também recursos públicos, o grau de preparação do concorrente dependerá da quantidade de recursos públicos de que se dispõe e de sua capacidade de usá-los na melhora da sua preparação. Os autores levam em consideração que os recursos públicos estão distribuídos de forma igualitária, havendo heterogeneidade entre os concorrentes quanto à capacidade de utilizar os recursos. Na terceira situação, os recursos privados agravam a desigualdade de oportunidade entre os participantes da corrida, dada a sua distribuição heterogênea (BARROS e MENDONÇA, 1995).

Os autores conseguem ainda observar os locais onde a desigualdade é gerada e transmitida a partir da constatação de que o mercado de trabalho tem discriminação e segmentação. A desigualdade de resultados constitui-se pelas diferenças individuais em preparação e pelas diferenças não relacionadas no período de preparação (BARROS e MENDONÇA, 1995) 
Especificamente sobre educação pública, Schultz (1963) argumenta que esse tipo de investimento em capital humano pode contribuir diretamente na redução da desigualdade de renda, uma vez que pode atingir parcela da população que não possui recursos para adquirir uma educação privada. Nessa mesma linha, Sylwester (2000) revela que caso os indivíduos sejam pobres o suficiente para não terem condições de estudarem, então cabe ao governo o papel de ofertante de educação pública como instrumento para, no longo prazo, reduzir a desigualdade de renda.

Nessa perspectiva, o debate centra na questão se a alocação de recursos escassos direcionada para gastos em educação pública pode refletir em efeitos positivos sobre a distribuição da renda. Se tal hipótese não for verdadeira, então a alocação de recursos em outras áreas pode vir a ser mais adequado para a população (SYLWESTER, 2000).

De maneira complementar, Jallade (1978) argumenta que a educação afeta a desigualdade de renda dependendo do tamanho do investimento feito no ensino e da taxa de retorno que se espera do investimento em educação. Logo, se os grupos socioeconômicos apresentam situações diferentes quanto ao acesso à educação, tal fato levanta importante indagação de caráter político na ação de políticas governamentais referente ao financiamento do ensino e a tributação de seus retornos que poderia contribuir para a redução da desigualdade de renda.

Ferreira (2000) nessa análise busca explicação a partir do processo de produção e educação. $\mathrm{O}$ argumento consiste no fato de que a distribuição de educação está relacionada às decisões políticas. Assim, deve-se considerar que políticas governamentais podem restringir os benefícios públicos para determinados grupos sociais de tal forma que as injustiças podem ser geradas na fase de distribuição de recursos públicos. Portanto, se o país enfrenta um equilíbrio Pareto-inferior de um sistema dinâmico onde as distribuições de educação, de riqueza e de poder político são determinadas simultaneamente, esse equilíbrio inferior, acaba gerando um círculo vicioso, pois parte de uma desigualdade de educação elevada, gerando altos níveis de desigualdade de renda e de riqueza e acaba conduzindo a uma distribuição desigual do poder político. Essa desigualdade política, por sua vez, resulta em uma desigualdade educacional, visto que os detentores do poder político não tem interesse na qualidade da educação pública, pois podem pagar pela educação privada, 
diferentemente dos mais pobres que não tem condições de financiar e frequentar uma escola privada, além de não possuir poder político para intervir nas decisões que se referem à melhora da qualidade das escolas públicas.

Em outra abordagem sobre educação, distinta da teoria do capital humano, Rawls (1971) considera a educação como um fator de grande importância dentro do sistema capitalista, pois não existe a equidade de oportunidades de trabalho entre os indivíduos. O autor hierarquiza sua concepção filosófica, dentro da qual coloca em primeiro lugar a questão do princípio da liberdade, em segundo o princípio da diferença e em último lugar, o princípio da igualdade de oportunidades. Sendo que em seu princípio da liberdade ressalta a liberdade de escolha, de expressão, de locomoção e o direito à liberdade.

No princípio da diferença, o autor argumenta que caso dois indivíduos partam de um mesmo ponto, e um deles, por seu talento e vocação pessoal acaba ficando mais rico que o outro, essa desigualdade pode ser tolerada, já que o indivíduo que tem mais talento acaba sendo mais eficiente do ponto de vista competitivo, e dessa forma a justificativa recai pelo sistema de concorrência. Por outro lado, no princípio da equidade de oportunidade, argumenta que os indivíduos devem ter as mesmas oportunidades, assim, é possível garantir uma sociedade justa.

Lin (2001) argumenta que é importante considerar no aspecto que tange a educação, ou capital social, a questão da estrutura social, e como essa estrutura se relaciona com a questão da educação. Enfatiza o autor que os processos de mudança social estão relacionados não apenas com o sistema educacional, mas com todo o aparato social de redistribuição de renda. Assim, contempla em sua análise também a questão das desigualdades no mercado de trabalho, aqui incluindo na análise inúmeras variáveis como gênero, etnia, etária, entre outros. Tais aspectos foram trabalhados em algumas pesquisas de natureza empírica que relacionam a questão do capital humano com a desigualdade de renda.

Lin (1999) afirma ainda que e a educação nesse sentido é vista como um dos capitais de um indivíduo e deve ser entendido como a captura de recursos da rede social na qual o individuo está inserido. Dessa forma, quando ao tratar de educação, a discussão é mais ampla ao envolver toda a rede social que envolve a questão. 
Woolcock e Narayan (2000) argumentam que é importante incorporar na analise diferentes níveis e dimensões, e reconhecer os pontos positivos e negativos que o capital social pode gerar. Essa visão, que incorpora diferentes níveis e dimensões do capital social, segundo os autores, é a visão que consegue melhores resultados do ponto de vista empírico. Nesse sentido, a educação deve ser vista dentro de um todo social, que pode ajudar a explicar as desigualdades de renda.

\subsection{Breve Revisão Empírica}

Os argumentos propostos por Schultz (1963) e Becker (1964), revelam ainda que a distribuição de renda é determinada pela forma como é distribuída a escolaridade de uma população. Assim, afirmam que há uma associação positiva entre desigualdade educacional e desigualdade de renda, ou seja, uma maior desigualdade na educação reflete em uma maior desigualdade de renda. Contudo, alertam que o efeito do aumento da escolaridade média pode ser negativo ou positivo, pois se trata de um efeito médio, cabendo analisar o retorno da educação de uma forma mais ampla como, por exemplo, para decis da população (GREGORIO e LEE, 2002).

De fato, a literatura apresenta algumas evidências favoráveis e outras contrárias ao efeito da educação sobre a desigualdade de renda, a partir da hipótese posta pela teoria do capital humano. Enquanto Becker e Chiscwick (1966) encontraram uma relação negativa entre nível médio de escolaridade e desigualdade de renda, outras evidências sugerem que tal relação não é válida como os trabalhos de $\operatorname{Ram}(1984,1989)$ ao apontar que não há significância estatística em suas estimativas.

Um número considerável de trabalhos realizados em diversos países em desenvolvimento identificou a educação como o mais importante atributo individual para explicar a desigualdade de renda. Adicionalmente, a ligação entre educação e desigualdade de renda é maior nos países em desenvolvimento do que nos já desenvolvidos (REIS e BARROS, 1990).

Dentre outros trabalhos podem ser destacadas as contribuições de Lam e Levison (1990), Glomm e Ravikumar (2003), Sylwester (2002) e Easterly e Rabelo (1993) que contribuem no entendimento da relação envolvendo a educação (seja de caráter público ou privado) e o nível de desigualdade de renda de um país. 
Gregorio e Lee (2002) estudam a relação entre educação e distribuição de renda a partir da metodologia de dados em painel para diversos países entre 1960 e 1990 . Os autores encontraram evidências de que o maior nível de escolaridade está associado a uma distribuição de renda mais igualitária.

\section{A RELAÇÃO ENTRE DESIGUALDADE E EDUCAÇÃO NO BRASIL}

\subsection{As origens do debate}

Langoni (1973) foi pioneiro ao ressaltar a importância da educação como fator explicativo para a desigualdade de renda brasileira. Com técnicas bastante avançadas para a época, mostrou que parte do aumento da desigualdade no Brasil entre 1960 e 1970 ocorreu devido ao aumento na demanda por trabalhadores qualificados vinculados à industrialização. Para tanto, utilizou dados disponíveis da época com foco de detectar as causas do aumento da desigualdade na distribuição de renda. A partir da amostra das rendas individuais extraídas do Censo Demográfico de 1970, analisou o perfil da distribuição da renda no Brasil. Uma das principais conclusões foi que a desigualdade na distribuição de renda dos grupos mais ricos é mais acentuada que os grupos mais pobres da população, também identificando que o grau de desigualdade aumentou no setor urbano.

Segundo Langoni (2005), a acentuada desigualdade de renda no Brasil ocorrida entre 1960 e 1970 teria como causa dois fatores. O primeiro é o papel da interação entre oferta e demanda no mercado de trabalho, na qual a acelerada expansão da atividade econômica conduziu a um aumento da demanda por mão de obra qualificada que, ligada a uma oferta relativamente inelástica no curto prazo, teve como consequência uma maior dispersão salarial entre os grupos de trabalhadores com diferentes níveis de qualificação. Além do mais, fatores relacionados à discriminação, segmentação e características individuais teriam mudado a composição da força de trabalho. Dentro desta última causa, a educação seria a variável chave na explicação do aumento da desigualdade de renda, enfatizando-se o papel de seus retornos no período eram plenamente justificáveis.

Langoni (2005) afirma que o crescimento resultante da época teria tido um viés tecnológico em virtude do processo de industrialização, o que, dentro 
desse contexto, teria provocado aumentos na demanda por mão de obra mais qualificada (especializada). Como não houve um acompanhamento recíproco da oferta, acabou-se gerando um desequilíbrio no mercado de trabalho em favor dos trabalhadores mais qualificados. Por outro lado, partindo da hipótese de Kuznets (1955)5, segundo a qual mudanças na composição do emprego e aumentos na desigualdade de renda resultam de um processo natural de desenvolvimento das economias capitalistas, Langoni afirma que os desequilíbrios estruturais à época vivenciadas pela economia brasileira eram plenamente justificáveis. Consequentemente, seria errôneo atribuir o aumento da desigualdade a perdas de bem-estar.

Fishlow (1972) com dados da década de 70 e 80 analisa a desigualdade de renda no Brasil. O autor identifica uma relação entre nível de educação e desigualdade de renda onde um menor acesso à educação e emprego em regiões rurais implica em níveis de renda menores e, por consequência, aumenta a desigualdade de renda em relação às regiões urbanas. Assim, a tese da compressão salarial do autor remete à ideia que o aumento da desigualdade de renda no Brasil esteve associado a uma política que refletia em redução dos salários médio e mínimo que, por consequência, reduzia a renda da população. Dada essa política, os ganhos eram concentrados nos setores urbanos o que refletia em efeitos perversos para as áreas rurais. Contudo, identificou um aumento superior na renda dos indivíduos com maior qualificação em comparação aos menos qualificados (TRINDADE, 2010).

De maneira complementar, Barros, Henrique e Mendonça (2002), observam que o valor de mercado da educação é particularmente influenciado pela distribuição da educação na população adulta e, portanto, reflete, em grande parte, a escassez relativa de trabalhadores qualificados. Sendo assim, um aumento na proporção de trabalhadores com maior nível de escolaridade poderia reduzir o valor de mercado da educação, tendo em vista que estaria sendo reduzida a escassez de trabalhadores qualificados.

Posteriormente ao debate da década de 70, outros autores retomam a discussão na década de 90. Reis e Barros (1991) relatam a importância da distribuição de educação para explicar a desigualdade. Os autores realizaram estudo entre regiões metropolitanas de São Paulo e Fortaleza. Em Fortaleza

\footnotetext{
A hipótese de Kuznets parte do argumento que em períodos iniciais de desenvolvimento a desigualdade tende a ser maior e, no longo prazo, tende a decair em virtude do aumento da renda associada à migração dos trabalhadores das regiões rurais para as urbanas.
} 
verificou-se um menor nível de desigualdade educacional, porém, o nível de desigualdade salarial foi em torno de $40 \%$ maior. Para justificar esse resultado, os autores observam que os diferenciais salariais associados à educação são maiores em Fortaleza, explicando $27 \%$ do diferencial entre as duas regiões. Segundo os autores, a menor desigualdade educacional e os maiores diferenciais salários são explicados pelo menor nível educacional em Fortaleza.

Para Bagolin e Porto Júnior (2003), no Brasil a desigualdade na escolarização da população é um dos principais fatores que explicam a desigualdade na distribuição de renda, pois um ano adicional de escolaridade no Brasil implica em valorização salarial elevada o que, aliado à escassez de mão de obra qualificada, contribui para concentração de renda pessoal no país. Nessa linha, Fernandes (2001) assegura que a criação de novas políticas de combate à desigualdade de rendimentos, necessariamente, deve levar em consideração políticas educacionais voltadas para a redução da desigualdade educacional. Em geral, o impacto direto das mudanças educacionais sobre a desigualdade de renda é que, tudo ou mais constante, um aumento (redução) na desigualdade em educação leva a aumento (redução) na desigualdade de rendimentos.

\subsection{Evidências empíricas para o Brasil}

Langoni (1973) estimou o impacto marginal de cada uma das variáveis qualitativas e alocativas sobre a renda com o objetivo de analisar a importância da educação. Para tanto, parte do método de variância através de regressões log-lineares tendo como variável dependente o log da renda que seria explicado por um conjunto de variáveis dummies representandas pelos indicadores de educação, idade, gênero, setor e região. O resultado obtido foi que as maiores diferenças de renda estão atreladas às diferenças de níveis de educação. A variável educação aumentou sua participação na explicação total da variância do log da renda entre 1960 e 1970 (51\% para $59 \%$ ) assumindo o maior valor explicativo, enquanto regiões e gênero perderam importância realtiva e a contribuição de atividade permaneceu praticamente constante durante todo período.

Velloso (1979) conclui em sua pesquisa que a desigualdade de renda é dependente das taxas médias de retorno da educação, dos ganhos da experiência ao longo do tempo, da desigualdade dos investimentos em educação, 
da idade do indivíduo e também da estabilidade do emprego do país. Tais fatores são parcialmente responsáveis pela desigualdade de renda do trabalho (salário). Portanto, quanto maior a desigualdade nos investimentos em educação (ou quanto mais elevado forem seus retornos médios), maior é a desigualdade de renda.

Reis e Barros (1990) mostram que mudanças na distribuição de educação por si só não reduz as diferenças regionais na desigualdade salarial. Isto só ocorre quando se computa o efeito estrutural, isto é, a mudança no perfil de salários. A desigualdade de salários é menor nas regiões metropolitanas do Sul e do Sudeste do que naquelas localizadas no Nordeste, não é porque a educação esteja mais bem distribuída nas primeiras, mas sim por talvez grupos relacionados sejam menores no Sul e Sudeste do que no Nordeste, dadas as mudanças na composição educacional da força de trabalho, que é decisiva para o desenho de uma política educacional que tenha como um de seus objetivos contribuírem para a redução da desigualdade salarial no país.

Se a desigualdade na escolarização da população é um dos principais fatores que explicam a desigualdade na distribuição de renda, então esse é um quadro grave quando se constata que a educação é um fator crucial para crescimento econômico de longo prazo entre as regiões e que um aumento nos anos médio de escolaridade tende a reduzir o diferencial na distribuição de renda educacional entre regiões no país, o que serviria, portanto, como fator adicional de crescimento de longo prazo. Além disso, não se pode, atribuir um padrão de U invertido, tal como versa a curva de Kuznets (1955), na evolução da desigualdade educacional, ou seja, ainda não terá alcançado um estágio de desenvolvimento onde o ensino universal, e em condições iguais, atingem todas as regiões do país. Essas observações deveriam ser levadas em consideração na elaboração de políticas públicas, as quais deveriam, por sua vez, perseguir metas mais amplas do que a universalização do acesso ao ensino para crianças (BAGOLIN e PORTO JÚNIOR, 2003).

Ramos e Vieira (2001) utilizam a metodologia de decomposição de alguns índices de desigualdade de rendimento no Brasil para as décadas de 1980 e 1990, com objetivo de captar a importância relativa de algumas fontes de dispersão de salário, inserindo as variáveis: a) educação e idade; b) posição na ocupação, região geográfica e setor de atividade econômica, para captar formas de inclusão no mercado de trabalho; c) gênero e cor, 
relacionados a possíveis discriminações. Constataram que a variável escolaridade tem um papel fundamental, sendo responsável pela desigualdade. As demais variáveis analisadas tiveram participação muito inferior na explicação da desigualdade de rendimentos no Brasil.

A pesquisa de Barros, Franco e Mendonça (2007) é motivada pelo processo educacional do Brasil que entre 1995 e 2005 sofreu uma expansão através do nível de escolaridade média do brasileiro, período esse concomitante com uma queda considerável no nível de desigualdade de renda em todas unidades federativas do país. Os autores argumentam que a queda na desigualdade de rendimentos do trabalho contribuiu fortemente na queda da desigualdade de renda, assim como a redução na desigualdade educacional. Por sua vez, a queda na desigualdade de rendimentos do trabalho se deve a uma redução nos diferenciais de remuneração por nível educacional.

Tabela 1. Evidências Empíricas a respeito da contribuição da educação para a desigualdade de renda no Brasil

\begin{tabular}{|l|l|l|l|l|}
\hline Autores & \multicolumn{1}{|c|}{ Método } & Período & \multicolumn{1}{c|}{ Nível de análise } & \multicolumn{1}{c|}{ Resultado } \\
\hline $\begin{array}{l}\text { Trindade } \\
(2010)\end{array}$ & $\begin{array}{l}\text { Séries de tempo } \\
\text { para relacionar a } \\
\text { desigualdade (Ín- } \\
\text { dice de Gini) em } \\
\text { função dos anos } \\
\text { de escolaridade }\end{array}$ & $\begin{array}{l}\text { Entre } \\
1995 \text { a } \\
2008\end{array}$ & Cinco regiões brasileiras & $\begin{array}{l}\text { Houve queda no Índice de } \\
\text { Gini associado ao aumento } \\
\text { dos anos de estudos em } \\
\text { todas as grandes regiões } \\
\text { do Brasil }\end{array}$ \\
\hline $\begin{array}{l}\text { Reis e } \\
\text { Barros } \\
(1990)\end{array}$ & $\begin{array}{l}\text { Analisar a relação } \\
\text { entre as distribui- } \\
\text { dones de trabalha- } \\
\text { dores segundo } \\
\text { seus salários e } \\
\text { níveis educacio- } \\
\text { nais }\end{array}$ & $\begin{array}{l}\text { Entre } \\
1976 \text { a } \\
1986\end{array}$ & $\begin{array}{l}\text { Nove regiões metropo- } \\
\text { rendanas brasileiras, com } \\
\text { masculino, com idade } \\
\text { entre 25 e 50 anos e carga } \\
\text { horária de 20 horas sema- } \\
\text { nais de trabalhada }\end{array}$ & $\begin{array}{l}\text { Mostra que cerca de 50\% } \\
\text { da desigualdade de salários } \\
\text { são explicados pela edu- } \\
\text { cação, há variações mais } \\
\text { altas nas regiões metropo- } \\
\text { litanas do nordeste onde } \\
\text { a diferença salarial é mais } \\
\text { elevada }\end{array}$ \\
\hline $\begin{array}{l}\text { Fernandes } \\
(2001)\end{array}$ & $\begin{array}{l}\text { Curvas de Lorenz } \\
\text { para investigar } \\
\text { a desigualdade } \\
\text { educacional em } \\
\text { relação à popu- } \\
\text { lação }\end{array}$ & $\begin{array}{l}\text { Entre } \\
1989 \text { a } \\
2001\end{array}$ & $\begin{array}{l}\text { Cinco regiões brasileiras } \\
\text { (exclusive pessoas da } \\
\text { área rural de Rondônia, } \\
\text { Acre, Amazonas, Rorai- } \\
\text { ma, Pará e Amapá) }\end{array}$ & $\begin{array}{l}\text { A região Nordeste é a mais } \\
\text { desigual e que a região } \\
\text { Sudeste. Nos estados de } \\
\text { São Paulo e da Bahia há } \\
\text { desigualdade na distribui- } \\
\text { ção de anos de estudo }\end{array}$ \\
\hline
\end{tabular}




\begin{tabular}{|c|c|c|c|c|}
\hline Autores & Método & Período & Nível de análise & Resultado \\
\hline $\begin{array}{l}\text { Bagolin } \\
\text { e Porto } \\
\text { Junior } \\
(2003)\end{array}$ & $\begin{array}{l}\text { Estimativa do } \\
\text { índice de Gini } \\
\text { Educacional }\end{array}$ & $\begin{array}{l}\text { Entre } \\
1983 \text { a } \\
2001\end{array}$ & $\begin{array}{l}\text { Distribuição de per- } \\
\text { centual da população } \\
\text { brasileira por } 5 \text { níveis de } \\
\text { escolaridade diferentes e } \\
\text { o índice de Gini educa- } \\
\text { cional para a população } \\
\text { acima de } 14 \text { anos no } \\
\text { Brasil }\end{array}$ & $\begin{array}{l}\text { Há diferença regional tanto } \\
\text { de níveis de escolaridade } \\
\text { como em desigualdade } \\
\text { educacional concentrados } \\
\text { nas regiões relativamente } \\
\text { pobres. Em alguns Estados, } \\
\text { como Alagoas, Roraima, } \\
\text { Piauí e Paraíba o índice de } \\
\text { Gini educacional são os } \\
\text { mais elevados do país. }\end{array}$ \\
\hline $\begin{array}{l}\text { Ramos } \\
(1991)\end{array}$ & $\begin{array}{l}\text { Decomposição es- } \\
\text { tática e dinâmica }\end{array}$ & $\begin{array}{l}\text { Entre } \\
1976 \text { a } \\
1885\end{array}$ & $\begin{array}{l}\text { Dados sobre homens } \\
\text { entre } 18 \text { e } 65 \text { anos, } \\
\text { ocupados em regiões bra- } \\
\text { sileiras, com rendimento } \\
\text { do trabalho positiva e } \\
\text { trabalhando } 20 \text { horas ou } \\
\text { mais por semana. }\end{array}$ & $\begin{array}{l}\text { A educação não apenas } \\
\text { oferece uma melhor isen- } \\
\text { ção no mercado de traba- } \\
\text { lho, mas também propor- } \\
\text { ciona algumas proteções de } \\
\text { curto prazo no desempenho } \\
\text { da economia. }\end{array}$ \\
\hline $\begin{array}{l}\text { Ramos } \\
\text { e Vieira } \\
(2001)\end{array}$ & $\begin{array}{l}\text { Análise relativa } \\
\text { da desigualdade } \\
\text { educacional e do } \\
\text { retorno à educação } \\
\text { sobre a variação } \\
\text { na desigualdade } \\
\text { de renda } \\
\end{array}$ & $\begin{array}{l}\text { Entre } \\
1981 \text { e } \\
1999\end{array}$ & $\begin{array}{l}\text { Dados sobre homens e } \\
\text { mulheres entre } 18 \text { e } 65 \\
\text { anos, que residem em } \\
\text { áreas urbanas e trabalham } \\
\text { pelo menos } 20 \text { horas por } \\
\text { semana }\end{array}$ & $\begin{array}{l}\text { A desigualdade de rendi- } \\
\text { mentos no Brasil, além de } \\
\text { ser uma das maiores no } \\
\text { mundo, permaneceu em } \\
\text { patamares elevados nos } \\
\text { últimos } 20 \text { anos }\end{array}$ \\
\hline $\begin{array}{l}\text { Ferreira } \\
\text { e Barros } \\
(1999)\end{array}$ & $\begin{array}{l}\text { Decomposição } \\
\text { baseada em micro- } \\
\text { simulação }\end{array}$ & $\begin{array}{l}\text { Entre os } \\
\text { anos de } \\
1976 \text { e } \\
1996\end{array}$ & $\begin{array}{l}\text { Areas urbanas no Brasil } \\
\text { (cerca de três quartos da } \\
\text { população). O modelo } \\
\text { geral se distingue em dois } \\
\text { sectores profissionais: os } \\
\text { assalariados e autoempre- } \\
\text { go em áreas urbanas }\end{array}$ & $\begin{array}{l}\text { Mostra que houve redução } \\
\text { do retorno médio à edu- } \\
\text { cação e aumento gene- } \\
\text { ralizado da qualificação } \\
\text { educacional dos indivíduos }\end{array}$ \\
\hline $\begin{array}{l}\text { Salvato, } \\
\text { Ferreira } \\
\text { e Duarte } \\
(2010)\end{array}$ & $\begin{array}{l}\text { Econometria se- } \\
\text { mi-paramétrica a } \\
\text { partir de densida- } \\
\text { des contrafactuais } \\
\text { de distribuição da } \\
\text { renda pela escola- } \\
\text { ridade }\end{array}$ & 1999 & $\begin{array}{l}\text { Regiões Nordeste e Su- } \\
\text { deste do Brasil e estados } \\
\text { selecionados (Ceará e } \\
\text { São Paulo) }\end{array}$ & $\begin{array}{l}\text { Entre } 12 \% \text { e } 36 \% \text { da } \\
\text { diferença de renda do } \\
\text { trabalho é explicada pelas } \\
\text { diferenças de escolaridade. } \\
\text { A reponderação pela esco- } \\
\text { laridade aumenta em } 55 \% \\
\text { a renda média das regiões } \\
\text { mais pobres }\end{array}$ \\
\hline
\end{tabular}

Fonte: Elaboração própria dos autores

O trabalho de Ferreira (2000) também vai nessa direção. O autor estuda o papel da educação no processo de redução da desigualdade de renda no 
Brasil e conclui que mais anos de estudos tendem a implicar em uma renda superior. Logo, a educação é um elemento importante para compreender a desigualdade de renda no país.

Com relação aos retornos à educação, Menezes Filho (2001) mostra que os trabalhadores com ensino fundamental concluído ganhavam em torno de três vezes mais que os analfabetos de 1997. De acordo com o estudo, a renda associada ao primeiro ano da faculdade era seis vezes maior que o rendimento médio dos analfabetos. $\mathrm{O}$ retorno associado ao primeiro ano do ensino superior também representava ganho salarial com relação a quem tinha apenas o ensino médio. Os trabalhadores com ensino superior completo, entre 15 e 16 anos de estudo, apresentavam rendimento salarial médio em torno de doze vezes superior ao grupo sem escolaridade. Essa diferença aumentava para dezesseis vezes para os indivíduos com mestrado.

Balassiano, Seabra e Lemos (2005) estudam a relação envolvendo escolaridade, salário e empregabilidade a partir de dados da Relação Anual de Informações Sociais (RAIS) na região metropolitana do Rio de Janeiro para o ano de 1999. Como resultado, identificam que o impacto da escolaridade no nível de salário e no emprego deve ser visto com ressalvas, pois há efeitos distintos. Por um lado, há um impacto positivo do aumento da escolaridade na renda do trabalhador, mas o efeito é significativo apenas para as faixas etárias de maior escolaridade, a partir do segundo grau. Então, o aumento da escolaridade resulta em incremento da renda apenas aos que possuem maior escolaridade.

A segunda constatação se refere ao fato que investimentos na educação não possuírem impacto significativo em termos de empregabilidade. $\mathrm{Ou}$ seja, não é possível afirmar que maior escolaridade implica em maior nível de emprego formal. Os autores atribuem esses resultados à base de dados utilizada na pesquisa.

Netto Junior e Figueiredo (2009) analisam as relações envolvendo distribuição de capital humano e desigualdade de renda para os estados no Brasil a partir de dados de 1986 até 2005. Os autores identificam que o nível de escolaridade da população se tornou menos disperso em relação à média. Resultado esse advindo de uma diminuição dos analfabetos e aumento do número de indivíduos com ensino médio s superior completo. Todavia, constatam que houve um aumento na dispersão da desigualdade 
do capital humano entre os estados brasileiros (exceto região Norte). Pela metodologia não-paramétrica constatam que há uma relação direta e não -linear entre desigualdade educacional e de renda nos períodos de 1997 até 1999 e de 2001 até 2005, caracterizados pela redução da desigualdade de renda associada à uma redução na desigualdade de capital humano.

Menezes Filho e Oliveira (2014), por sua vez, analisam o papel da educação na queda da desigualdade de renda no Brasil com dados para o período de 1992 à 2009. Através de simulações para identificar o efeito da renda do trabalho a das transferências de renda sobre a desigualdade de renda e também da contribuição da educação no crescimento da renda do trabalho por meio de regressões mincerianas, os autores concluem que a educação contribuiu com algo em torno de $40 \%$ e $25 \%$ para a queda da desigualdade de renda no mercado de trabalho e na renda familiar, respectivamente, para o período entre 2001 e 2009.

No Brasil, tanto a desigualdade educacional quanto os retornos à educação são elevados e influencia negativamente a desigualdade de rendimentos. Logo, políticas centradas na expansão educacional como foco para a redução da desigualdade de renda no país tendem a ser eficientes (TRINDADE, 2010).

\section{BASE DE DADOS E METODOLOGIA}

Dada a relevância apresentada pela literatura sobre o estudo envolvendo os efeitos da educação sobre a desigualdade de renda e pelo fato das evidências empíricas que envolvem essa relação não apresentarem consenso, a presente pesquisa propõe uma análise empírica relativa aos Estados brasileiros em período após a implantação do Plano Real. Para tanto, parte de uma metodologia de dados em painel, distinta dos métodos geralmente empregados para pesquisas deste tema relativas ao Brasil.

Os dados utilizados na análise se referem a todos os estados do Brasil inclusive o Distrito Federal, durante o período entre 1995 e $2009^{6}$. Os dados de desigualdade de renda (índice de Gini), renda domiciliar per capita e

${ }^{6}$ A fonte de dados secundária, IPEADATA, ainda não disponibilizou os dados da renda familiar per capita e anos médios de estudo para o ano de 2010 , sendo assim o período do estudo se limita até o ano de 2009. 
média de anos de estudo para pessoas com 25 anos ou mais de idade ${ }^{7}$ foram obtidos junto ao banco de dados do Instituto de Pesquisa Econômica Aplicada (Ipeadata) e ao Instituto Brasileiro de Geografia e Estatística (IBGE).

Todas as variáveis, com exceção do índice de Gini, são apresentadas em logaritmo. A Tabela 2 mostra o resumo das abreviações das variáveis do modelo para facilitar a leitura posterior das parametrizações.

Tabela 2 - Lista das Variáveis do Modelo

\begin{tabular}{l|l}
\hline \multicolumn{1}{c|}{ Variáveis } & \multicolumn{1}{c}{ Notação } \\
\hline Índice de Gini & GINI \\
Renda domiciliar per capita & LRENDA \\
Anos de estudo para pessoas com 25 anos ou mais & LESTUDO \\
\hline
\end{tabular}

Fonte: Elaboração própria dos autores

No que tange a metodologia, segundo Salvato et al. (2006), o método de cross-section apresenta limitações ao ignorar diferenças históricas específicas de cada estado em suas respectivas trajetórias de crescimento. Esta omissão poderia gerar erros nos resultados estimados. Portanto, como alternativa, será utilizado o procedimento de dados em painel, uma vez que esse considera as particularidades contidas em cada unidade federativa.

O modelo de dados em painel é indicado quando estão disponíveis observações longitudinais, isto é, por indivíduos num espaço de tempo, o que fornece informações a respeito de possíveis heterogeneidades individuais ${ }^{8}$. De acordo com Greene (2002), o método é bastante utilizado para investigar mudanças estruturais e dinâmicas de transição.

Este modelo possui algumas vantagens importantes. Dentre elas, Hsiao (2003) destaca algumas como a oportunidade de redução em problemas de colinearidade e de variáveis omitidas e aumento dos graus de liberdade, o que permite que a dinâmica intertemporal e a individualidade das variáveis analisadas possam ser mais bem controladas, no que se refere aos efeitos da omissão de outras variáveis.

\footnotetext{
A média de anos de estudo de pessoas com 25 anos ou mais de idade, segundo o IPEADATA, é dada pela razão entre o somatório do número de anos de estudo completados pelas pessoas que tem 25 ou mais anos de idade e o número de pessoas nessa faixa etária.

8 Ver Cameron e Trivedi (2005) para uma maior compreensão das vantagens que advém do uso dos modelos que captam a heterogeneidade dos indivíduos.
} 
A estimativa por dados em painel considera as observações em distintos instantes de tempo. Para o presente caso sua forma funcional é do tipo:

$$
G I N I_{i t}=\alpha+\beta_{1} \operatorname{LRENDA}_{i t}+\beta_{2} \operatorname{LESTUDO}_{i t}+\varepsilon_{i t}
$$

onde, $t$ é o indicador do tempo e $i$ se refere à unidade federativa analisada. O método de dados em painel pode ser estimado tanto por efeitos fixos (FE) quanto por efeitos aleatórios (RE). Para efeitos fixos a estimativa controla as variáveis omitidas quando essas oscilam entre observações, contudo são constantes ao longo do tempo. Sua forma funcional é do tipo:

$$
G I N I_{i t}=\beta_{1} \operatorname{LRENDA}_{i t}+\beta_{2} \operatorname{LESTUDO}_{i t}+\chi_{i}+\varepsilon_{i t}
$$

onde $\chi_{i}=\alpha+\beta_{3} \gamma_{i}$ e a variável omitida $\gamma_{i}$ capta os fatores que não são observados e variam entre os estados, mas que são constantes ao longo do tempo. Como exemplos de variáveis omitidas podem ser citadas: características geográficas inerentes a cada estado, o nível educacional da população ou mesmo atributos específicos de setores econômicos.

Por sua vez, $\varepsilon_{i t}$ representa o erro idiossincrático, termo esse que indica os fatores que oscilam ao longo do tempo, mas que não são observados. É possível observar ainda que no modelo de efeitos fixos os erros não podem apresentar correlação ao longo do tempo e entre estados, sendo condicionais aos regressores. Assim, busca-se estimar $\gamma_{i}$, a variável não observada que é constante ao longo do tempo, mas que varia entre as unidades federativas.

Já na estimativa de efeitos aleatórios, as variáveis oscilam ao longo do tempo, mas são constantes entre estados. A especificação é do tipo:

$$
\operatorname{LGINI}_{i t}=\alpha+\beta_{1} \operatorname{LRENDA}_{i t}+\beta_{2} \operatorname{LESTUDO}_{i t}+\beta_{3} \gamma_{i}+\beta_{4} \delta_{t}+\varepsilon_{i t}
$$

onde, $\delta_{t}$ representa a variável omitida que varia ao longo do tempo, mas que é constante entre estados. Se $\delta_{t}$ for correlacionado com $\triangle L R E N D A_{i t}$, então haverá viés da variável omitida na estimativa. Em contrapartida, se $E\left(u_{i t} \mid \delta_{t}\right) \neq 0$, então a estimativa via OLS (Ordinary least squares) deixa de ser eficiente, sendo recomendado o método GLS (genralized least squares) por apresentar parâmetros mais eficientes. 
A escolha entre o modelo de efeitos fixos e efeitos aleatórios é dada pelo teste de Hausman. A hipótese nula do teste é de que não há correlação entre o erro idiossincrático e as variáveis independentes no modelo de efeitos aleatórios. Portanto, se não rejeitada a hipótese nula, o modelo de efeitos aleatórios é consistente e as diferenças de todos os coeficientes não é sistemática. Em contrapartida, se rejeitada indica que o modelo de efeitos fixos é mais consistente. ${ }^{9}$

Assim como no caso de estudos de séries temporais, deve-se verificar se as séries utilizadas são estacionárias. Segundo Morettin e Toloi (2004), um processo estacionário ocorre numa série temporal quando ele se desenvolve no tempo, de modo que a escolha da amostra temporal, ou janela temporal não seja importante. Caso a estacionariedade não seja verificada, ou seja, havendo a presença de raiz unitária, pode-se ter o problema de regressão espúria.

Entre os testes de estacionariedade mais usuais na literatura de dados em painel figuram o teste proposto por Levin et al (2002) e teste proposto por Im et al (2003). A diferença entre esses dois testes é que o segundo permite a existência de um processo individual de raiz unitária onde os parâmetros de persistência podem variar para cada unidade, enquanto que o primeiro assume a existência de um processo de raiz unitária comum. A hipótese nula dos testes é a de que a série seja integrada de primeira ordem I(1), contra a hipótese de que a série seja estacionária.

\section{RESULTADOS EMPÍRICOS}

Como já ressaltado, os dados que compõem a amostra desse estudo são referentes ao índice de Gini, representando a desigualdade de renda, a renda domiciliar per capita, e por fim, anos de estudo para pessoas com 25 anos ou mais, sendo essa usada como proxy para a variável educação. A seguir são apresentadas as estatísticas descritivas: ${ }^{10}$

\footnotetext{
Alguns autores utilizam a denominação de within para a estimativa de efeitos fixos e FGLS (feasible generalized least squares) - pondera-se as variáveis pelo desvio-padrão, resultando em resíduos mais consistentes - para efeitos aleatórios.

${ }^{10}$ Os resultados foram obtidos através do software Stata, versão 12.
} 
Tabela 3 - Estatística descritiva das variáveis analisadas

\begin{tabular}{l|c|c|c|c}
\hline Variáveis & Média & Desvio-Padrão & Valor Mínimo & Valor Máximo \\
\hline GINI & 0,5658 & 0,0422777 & 0,426 & 0,68 \\
LRENDA & 615,7565 & $2,522.282$ & 218,27 & $1.744,97$ \\
LESTUDO & 5,804 & 1,207 & 3,2 & 9,6 \\
\hline
\end{tabular}

Fonte: Elaboração própria dos autores a partir dos dados do IPEADATA

$\mathrm{Na}$ amostra utilizada, o estado que apresentou o menor índice de Gini foi Roraima registrando um valor de 0,426 (1995). Em sentido oposto, o estado com maior valor foi Amazonas (2000) com o valor de 0,68. Já o valor médio desse índice foi de 0,5658 .

Quanto ao nível de renda domiciliar, o valor médio registrado é de R\$ 615,7565 e o Maranhão foi o estado que indicou o menor valor na amostra (2000). Na outra mão, o Distrito Federal registrou o maior valor dessa variável $(\mathrm{R} \$ 1.744,97)$ parao ano de 2009.

No que tange aos dados referentes aos anos de estudos, tem-se que o valor médio registrado é de 5,8 anos. O menor valor dessa série foi 3,2 registrado no estado de Maranhão (1995), enquanto que o maior valor pertence ao Distrito Federal de 9,6 anos em média (2009).

Recorreu-se a técnicas de dados em painel, conforme já mencionado, para obter as relações entre a desigualdade e as variveis educação e renda. Primeiramente, se fez necessário a realização dos testes de estacionariedades de Levin et al (2002) e Im et al (2003) com tendência e constante. Os resultados são dispostos na Tabela 4 .

De acordo com os resultados apresentados na Tabela 4, pode ser constatado que para todas as variáveis é rejeitado a hipótese nula, o que indica que as séries empregadas nesse estudo são estacionárias, o que viabiliza estimações econométricas não espúrias.

Como já ressaltado, a literatura sobre desigualdade aponta que tanto a renda, quando a educação possuem relação inversa com a desigualdade, tal como sugerem Velloso (1979), Barros, Franco e Mendonça (2007), Menezes Filho e Oliveira (2014), entre outros. Sendo assim, espera-se uma relação negativa entre tais variáveis e a desigualdade no Brasil. A 
Tabela 4 - Testes de Estacionariedade

\begin{tabular}{lcc}
\hline Variáveis & Levin, Lin \& Chu & In, Pesaron, Shin W-stat \\
\hline GINI & $-4,6632 *$ & $-7,4201 *$ \\
LRENDA & $-2,4637 *$ & $-3,291 *$ \\
LESTUDO & $-7,9954 *$ & $-7,5323 *$ \\
\hline $\begin{array}{l}\text { Notas: } \\
\text { 1- } \quad \text { 1\% de significância; **5\% de significância; ***10\% de } \\
\text { significância }\end{array}$ \\
\hline
\end{tabular}

Fonte: Elaboração própria dos autores

Tabela 5 apresenta os resultados da correlação de Pearson.

Tabela 5 - Correlação entre Índice de Gini com Renda e Anos de Estudos

\begin{tabular}{ccc|ccc|ccc}
\hline UF & LRENDA & LEST & UF & LRENDA & LEST & UF & LRENDA & LEST \\
\hline AC & $-0,0552$ & $-0,217$ & MA & $-0,5085$ & $-0,6259^{*}$ & RJ & $-0,6175^{*}$ & $-0,492$ \\
AL & 0,0532 & $-0,4375$ & MG & $-0,6977^{*}$ & $-0,7355^{*}$ & RN & $-0,5635^{*}$ & $-0,8078^{*}$ \\
AM & $-0,2665$ & $-0,7717^{*}$ & MS & $-0,4943$ & $-0,5669^{*}$ & RO & 0,4151 & $-0,7464^{*}$ \\
AP & $-0,6202^{*}$ & $-0,5698^{*}$ & MT & $-0,4064$ & $-0,6556^{*}$ & RR & $-0,7802^{*}$ & 0,037 \\
BA & $-0,7146^{*}$ & $-0,6758^{*}$ & PA & $-0,4075$ & $-0,7310^{*}$ & RS & $-0,7989^{*}$ & $-0,8351^{*}$ \\
CE & $-0,8131^{*}$ & $-0,8587^{*}$ & PB & $-0,188$ & $-0,3329$ & SC & $-0,7377^{*}$ & $-0,7434^{*}$ \\
DF & $-0,136$ & 0,2099 & PE & $-0,0122$ & $-0,4745$ & SE & $-0,3457$ & $-0,6908^{*}$ \\
ES & $-0,8500^{*}$ & $-0,7571^{*}$ & PI & $-0,5508^{*}$ & $-0,5174 *$ & SP & $-0,6346^{*}$ & $-0,6323^{*}$ \\
GO & $-0,6968^{*}$ & $-0,7505^{*}$ & PR & $-0,9005^{*}$ & $-0,7042 *$ & TO & $-0,7428^{*}$ & $-0,8463^{*}$ \\
\hline
\end{tabular}

Notas:

1- * $1 \%$ de significância; $* * 5 \%$ de significância; $* * * 10 \%$ de significância

Fonte: Elaboração própria dos autores

Os resultados indicam que a maioria dos estados apresentam os coeficientes de correlação como o esperado, ou seja, negativos e estatisticamente diferentes de zero. De forma específica, tem-se que para os estados do Acre, Alagoas, Paraíba, Pernambuco e o Distrito Federal, tanto os coeficientes para a renda quanto anos de estudos são estatisticamente insignificantes. Outro ponto que deve ser ressaltado é o fato de que os coeficientes relacionados 
à variável anos de estudos com o Índice de Gini mostraram ser estatisticamente significantes, distintamente do que os que retrata a correlação entre a renda e o Índice de Gini. Os estados que apresentaram uma correlação forte entre a proxy da educação e desigualdade foram Ceará, Rio Grande do Norte, Rio Grande do Sul e Tocantins.

Após a verificação de significante correlação entre as variáveis, foram estimados modelos com efeitos fixos e com efeitos aleatórios. A Tabela 6 ilustra os resultados obtidos e segundo os testes F e de Wald pode-se rejeitar a hipótese nula de não significância conjunta dos parâmetros das varáveis explicativas.

Tabela 6 - Modelo Econométrico

\begin{tabular}{|c|c|c|c|c|c|c|}
\hline \multirow[b]{2}{*}{$\begin{array}{c}\text { Variáveis } \\
\text { independentes }\end{array}$} & \multicolumn{3}{|c|}{ Efeitos Fixos } & \multicolumn{3}{|c|}{ Efeitos Aleatórios } \\
\hline & Coeficientes & Erro-Padrão & $\mathbf{T}$ & Coeficientes & Erro-Padrão & $\mathbf{T}$ \\
\hline LRENDA & $-0,0459042 *$ & 0,0092031 & $-4,99$ & $-0,0314563^{*}$ & 0,0088197 & $-3,57$ \\
\hline LESTUDO & $-0,1250713 *$ & 0,0135596 & $-9,22$ & $-0,1203781 *$ & 0,0135897 & $-8,86$ \\
\hline CONS & $1,074406^{*}$ & 0,0492828 & 21,8 & $0,974538 *$ & 0,0456944 & 21,33 \\
\hline \multicolumn{4}{|l|}{$\mathrm{R} 2=0,3848$} & \multicolumn{3}{|l|}{$\mathrm{R} 2=0,3827$} \\
\hline \multicolumn{4}{|l|}{$\mathrm{F} \quad 17,76 \quad(0,0000)$} & \multicolumn{3}{|c|}{ Wald $210,08 \quad(0,0000)$} \\
\hline Teste Hausman & $63(0.9394)$ & & & & & \\
\hline
\end{tabular}

Notas:

1- * $* 1 \%$ de significância; $* * 5 \%$ de significância; $* * * 10 \%$ de significância

2- P-valor entre parênteses

Fonte: Elaboração própria dos autores

De acordo com o resultado do modelo estimado por efeitos fixos, tem-se que todas variáveis explicativas foram estatiticamente significantes, ao nível de $1 \%$. Sendo assim, para essa amostra de dados o resultado encontrado sinaliza que tanto a renda quanto a educação afetam o Índice de Gini. Para ambas as variáveis os sinais encontrados dos parâmetros corraboram com a 
literatura teórica da teoria do capital humano de que o aumento da educação pode contribuir na redução da desigualdade e renda e vai na mesma linha de diversas evidências empíricas sobre o tema, tais como os trabalhos de Velloso (1979), Salvato, Ferreira e Duarte (2010), Trindade (2010), Menezes Filho e Oliveira (2014), Netto Junior e Figueiredo (2009) e Balassiano, Seabra e Lemos (2005), apesar dessas pesquisas apresentarem níveis de análise distintos para o Brasil e outras metodologias.

Os resultados obtidos por efeitos aleatórios são semelhantes daqueles obtidos ao empregar o modelo com efeitos fixos. A proxy para renda e educação utilizada no modelo foram ambas foram estatisticamente significante a nível de $1 \%$ e com sinais esperados. As evidências obtidas apontam para a mesma direção já formulada por Velloso (1979) que argumenta que a expansão da oferta de educação, seja por meio de investimentos público ou privado, ou mesmo a redução das desigualdades nos níveis de educação em uma determinada região pode implicar em uma medida, dentre tantas outras para tornar a distribuição da renda mais igualitária.

Para verificar qual dos modelos (efeito fixo ou aleatório) é mais eficiente aplica-se o teste de Hausman. A hipótese nula é de que não há correlação entre o erro idiossincrático e as variáveis explicativas no modelo de efeitos fixos, sendo esse mais consistente e não havendo diferenças sistemáticas nos coeficientes. De acordo com o resultado obtido, percebese um melhor ajustamento do estimador de efeitos fixos, uma vez que se rejeita a hipótese nula de não correlação entre os efeitos específicos e as variáveis explicativas. Sendo assim, o teste aponta para a conveniência da estimação do modelo de efeito fixo, o que sugere que existem diferenças estaduais quer a nível de ambiente político, institucional e econômico que podem interferir nesse processo.

Em suma, os resultados encontrados sugerem que a renda e a educação estão em consonância com o que aponta a literatura. Fica claro pela magnitude dos parâmetros que existem outros fatores essenciais que explicam a desigualdade de renda. Assim, tal como sugere a literatura sobre desigualdade, a identificação dos determinantes da desigualdade de renda não renda não é trivial.

Dentre outros fatores, a literatura empírica destaca a pobreza, a ausência de programas de transferência de renda voltados aos mais pobres, as 
desigualdades educacionais e salariais e sistemas políticos que não promovam políticas de igualdade como fatores que podem auxiliar nesse sentido. Adicionalmente, Salvato, Ferreira e Duarte (2010), elencam outros fatores que podem contribuir nesse entendimento, tal como fatores institucionais, a longevidade da população, questões de cunho histórico, infra-estrutura disponível, entre outros.

\section{CONCLUSÃO}

Segundo abordagem da teoria do capital humano, o aumento do nível educacional de uma população pode implicar em ganhos de produtividade do fator trabalho e refletir aumentos, por sua vez, nas rendas médias. Tal condição poderia gerar uma condição mais igualitária em termos de distribuição de renda.

A partir da implantação do plano Real, em 1995, é possível identificar que o Brasil passou a indicar aumentos no nível de renda média, melhora nos índices de frequência e escolaridade e uma acentuada queda no índice de desigualdade de renda, distintamente de período anteriores.

Diante desse contexto, o objetivo da pesquisa foi pautado em compreender a relação envolvendo educação e desigualdade de renda para o Brasil a partir de dados para o período de 1995 e 2009, por meio da metodologia de dados em painel. Adicionalmente, foi também utilizada para as estimativas a variável de renda domiciliar.

De acordo com o resultado do modelo estimado por efeitos fixos, tem-se que todas variáveis explicativas foram estatiticamente significante, ao nível de $1 \%$ e com os sinais esperados como aponta a literatura teórica e empírica sobre o assunto. Sendo assim, para essa amostra de dados o resultado encontrado sinaliza que tanto a renda quanto a educação afetam negativamente o Índice de Gini. Pela magnitude dos parâmetros econtrados pode ser obervado que a educação tem um imapcto maior no Índice de Gini em relação a renda e que existem outros fatores essenciais que explicam a desigualdade de renda no Brasil, fato que constitui como principal limitação do trabalho. Segundo a literatura, fatore institucionais, a longevidade da população, questões de cunho histórico, podem ser fatores que contribuem no entendimento dessa questão. 
Quanto aos resultados das estimações, tem-se que o teste Hasuman indicou que o modelo de efeitos fixos é preferível ao modelo de efeitos aleatórios, sinalizando que diferenças estaduais, tal como fatores históricos, por exemplo, são relevantes para explicar a desigualdade de renda entre as unidades Federativas do Brasil. A ausência de correlação dos resultados obtidos indica que a educação é importante para compreender a desigualdade de renda, pois com o aumento no nível de escolaridade tende a haver uma redução na desigualdade de renda (a distribuição da renda da população se torna mais igualitária), hipótese essa identificada em diversos trabalhos relacionados ao tema.

\section{RELATIONSHIP BETWEEN INEQUALITY AND EDUCATION IN BRAZIL: AN ESTIMATE OF PANEL DATA (1995-2009)}

\section{Abstract:}

The literature dealing with human capital argues that education has a positive effect on labor productivity factor, which results in an increase in the level of wages. Indeed, ceteris paribus, increases in schooling reflected in the increase of the product and may contribute to the reduction in inequality. In this context, the aim of this study is to investigate whether there is an inverse relationship between inequality of income and education for the Federative Units of Brazil between 1995 and 2009. Therefore, part of the econometric methodology of panel data with indicators of income inequality and education, in addition to per capita household income. The results go in the same direction on the subject several evidences to indicate that the variables income and education are inversely correlated with income inequality, with statistically significant results.

Keywords: Education; Income and Inequality.

JEL Classification: I24, D63. 


\section{REFERÊNCIAS}

BAGOLIN, Izete Pengo; PÔRTO JÚNIOR, Sabino da Silva. A desigualdade da distribuição da educação e crescimento no Brasil: índice de Gini e anos de escolaridade. Estudos do CEPE (UNISC), Santa Cruz do Sul - RS, v. 18, p. 7-31, 2003.

BALASSIANO, Moisés; SEABRA, Alexandre Alves de; LEMOS, Ana Heloisa. Escolaridade, salários e empregabilidade: Tem razão a teoria do capital humano? Revista de Administração Contemporânea, v. 9, n.4, p. 31-52, out/dez, 2005.

BARROS, Ricardo Paes de; FRANCO, Samuel; MENDONÇA, Rosane. A recente queda da desigualdade e o acelerado progresso educacional brasileiro da última década. Rio de Janeiro, IPEA, Texto para Discussão, $n^{\circ} 1304$, setembro/2007.

BARROS, Ricardo Paes de; HENRIQUES, Ricardo; MENDONÇA Rosane. Pelo fim das décadas perdidas: Educação e desenvolvimento sustentado no Brasil. Rio de Janeiro, IPEA, Texto para Discussão, n 857, jan/2002.

BARROS, Ricardo Paes de; MENDONÇA Rosane Silva Pinto de. Os determinantes da desigualdade no Brasil, Rio de Janeiro, IPEA, Texto para Discussão, n 377, jul/1995.

BECKER, Gary. Human Capital: A Theoretical and Empirical Analysis With Special Reference to Education. Chicago: The University of Chicago Press, 1964.

BECKER, Gary; CHISWICK, Barry. Education and the distribution of earnings. American Economic Review. Papers and proceedings, n. 56, p. 358-369, 1966.

CAMERON, A. Colin; TRIVEDI, Pravin K. Microeconometrics methods and applications. Cambridge Press, 2005. 
EASTERLY, William; REBELO, Sergio. Fiscal policy and economic growth: An empirical investigation. Journal of Monetary Economics, v. 32, p. 417-455, 1993.

FERNANDES, Cláudia Monteiro. Desigualdade de rendimentos e educação no Brasil: alguns indicadores de diferenças regionais. Revista Econômica, v.3, n.2, p 231-250, dez/2001

FERREIRA, Francisco H. G. Os determinantes da desigualdade de renda no Brasil: luta de classe ou heterogeneidade educacional? Rio de Janeiro: PUC - Departamento de Economia (Texto para Discussão, n 415), fev/2000.

FERREIRA, Francisco H. G; BARROS, Ricardo Paes de. The slippery slope: explaining the increase in extreme poverty in urban Brazil, 1976-1996. Revista de Econometria, Rio de Janeiro, v. 19, n. 2, p. 211-296, nov. 1999.

FRANÇA, Gilson Nardo; GASPARINI, Carlos Eduardo ; LOUREIRO, Paulo Roberto Amorim . Relação entre Escolaridade e Renda na Década de 1990. Encontro Regional de Economia, 2005, Fortaleza. Anais do X Encontro Regional de Economia. Fortaleza: Banco do Nordeste do Brasil, 2005.

FISHLOW, Albert. Brazilian size distribution of income. American Economic Review. Nashville, v. 62, n.2, 1972.

GLOMM, Gerhard; RAVIKUMAR, B. Public education and income inequality. European Journal of Political Economy, v. 19, p. 289-300, 2003.

GREENE, William H. Econometric analysis. 5th ed. Upper Saddle River, New Jersey: Prentice Hall, 2002.

GREGORIO, José de; LEE, Jonh-Wha. Education and income inequality: New evidence from cross-croutry data. Review of Income and Wealth. Series 48, n. 3, setembro/2002.

HSIAO, Cheng. Analysis of Panel Data. 2 ed. New York: Cambridge University Press. 2003. 
IM, Kyung So; PESARAN, M. Hashem; SHIN, Yongcheol. Testing for Unit Roots in Heterogeneous Panels. Journal of Econometrics, v. 115, p. 53-74, 2003

INSTITUTO DE PESQUISA ECONÔMICA APLICADA. Disponível em $<$ www.ipea.gov.br $>$ Acesso em 23 set 2013.

JALLADE, Jean Pierre. Educação de primeiro grau e desigualdade de renda no Brasil: perspectiva a longo prazo. Revista e Pesquisa Planejamento Econômico, Rio de Janeiro, v. 8, n. 2, p. 561-596, dez/ 1978.

KUZNETS, Simon. Economic growth and income inequality. American Economic Review, v. 45, p. 1-28, 1955.

LAM, David; LEVISON, Deborah. Age, experience, and schooling: Decomposing earnings inequality in the United States and Brazil. Sociological Inquiry, vl. 62, n. 2, Spring, 1992.

LANGONI, Carlos. G. Distribuição da Renda e Desenvolvimento Econômico do Brasil. Rio de Janeiro, Expresso e Cultura, 1973.

LANGONI, Carlos. G. Distribuição de Renda e Desenvolvimento Econômico no Brasil. 3. Ed. Rio de Janeiro: FGV, 2005.

LEVIN, A.; LIN, C.F.; CHU, C. S. J. Unit Root Tests in Panel Data: Asymptotic AN Finite sample Properties. Journal of Econometrics, v. 108, p. 1-24, 2002.

LIN, Nan. Building a network theory of social capital. Connections, v. 22, p. 28-51, 1999.

LIN, Nan. Social capital: A theory of social structure and action. Cambridge: Cambridge University Press, 2001.

MENEZES FILHO, Naercio A. A evolução da educação na Brasil e o seu impacto no mercado de trabalho. Departamento de Economia - Uni- 
versidade de São Paulo (Artigo preparado para o Instituto Futuro Brasil), março de 2001.

MENEZES FILHO, Naercio, A.; OLIVEIRA, Alison Pablo de. A contribuição da educação para a queda na desigualdade de renda per capita no Brasil. Centro de Políticas Públicas - Insper, Policy Paper n. 9, janeiro, 2014.

MORETTIN, Pedro Alberto.; TOLOI, Clelia M. C. Análise de séries temporais. Edgard Blucher, Associação Brasileira de Estatística ABE - Projeto Fisher, 2004.

NETTO JUNIOR, José Luis da Silva; FIGUEIREDO, Erik Alencar de. Distribuição de capital humano e desigualdade de renda: Mobilidade intergeracional educacional e mobilidade de renda no Brasil. Economia e Desenvolvimento. Recife, v. 8, n.1, 2009.

PEREIRA, Dilson Jose de Sena. Diferenças de escolaridade e rendimento do trabalho nas regiões nordeste e sudeste do Brasil. Dissertarão (Mestrado Economia Aplicada), Escola Superior de Agricultura Luiz de Queiroz, Piracicaba, 2001.

RAM, Rati. Population increase, economic growth, educational inequality, and income distribution: some recent evidence. Journal of Development Economics, n. 14, p. 419-428, abril/1984.

RAM, Rati. Can educational expansion reduce income inequality in lessdevelopment countries? Economics of Education Review, v. 8, n.2, p. 185-189, 1989.

RAMOS, Lauro. Desigualdade de renda e crescimento econômico no Brasil. Rio de Janeiro: IPEA, Texto para Discussão, n. 213, 1991.

RAMOS, Lauro; VIEIRA Maria Lucia. Desigualdade de rendimentos no Brasil nas décadas de 80 e 90: evolução e principais determinantes. Rio de Janeiro: IPEA, Texto para Discussão, n. 803, 2001. 
RAWLS, John. A theory of Justice. Cambridge, MA: Belknap Press, 1971.

REIS, José Guilherme Almeida; BARROS, Ricardo Paes de. Desigualdade Salarial e Distribuição de Educação: A Evolução das Diferenças Regionais no Brasil. Pesquisa e Planejamento Econômico, Rio de Janeiro, v.20, n.3, p.415-478, dez. 1990.

REIS, José Guilherme Almeida; BARROS, Ricardo Paes de. Wage inequality and the distribution of education: a study of the evolution of brazilian regional differences in inequality. Journal of Development Economics, v 36, 1991.

SALVATO, Márcio Antônio; ALVARENGA, Patrícia SILVA; FRANÇA, Cristina Soares; ARAUJO JUNIOR, Ari Francisco de. Crescimento e desigualdade: Evidências da curva de Kuznets para os municípios de Minas Gerais - 1991/2000. Ibmec - MG, Working Paper, n. 33, 2006. Disponível em < http://www.ceaee.ibmecmg.br/wp/wp33.pdf>, acesso em 10 de mar, 2014.

SALVATO, Márcio Antônio; FERREIRA, Pedro Cavalcanti Gomes; Duarte, Angelo José Mont'Alverne. O impacto da escolaridade sobre a distribuição de renda. Estudos Econômicos. São Paulo, v. 40, n. 4, p. 753-791, outubrodezembro, 2010.

SCHULTZ, Théodore W. The economic value of education. New York: Columbia University Press, 1963

SYLWESTER, Kevin. Income inequality, education expenditures, and growth. Journal of Development Economics, v. 63, p. 379-398, 2000.

SYLWESTER, Kevin. Can education expanditures reduce income inequality? Economics of Education Review, n. 21, p. 43-52, 2002.

TRINDADE, Flávia Valim da. Distribuição de renda e educação: A discussão brasileira a partir da década de 70. Monografia (Bacharelado 
em Ciências Econômicas), Universidade Federal do Rio Grande do Sul departamento de ciências econômicas, Porto Alegre, 2010.

VELLOSO, Jacques R. Educação e desigualdade de renda urbana no Brasil: 1960/80. Pesquisa e Planejamento Econômico, Rio de Janeiro, v. 9, n. 3, p. 661-718, dez. 1979.

WOOLCOOCK, Michael; NARAYAN, Deepa. Social capital: Implications for development theory, research and policy. World Bank Research Observer, n. 15, v. 2, p. 225-250, 2000. 